Article original

\title{
Hyper-réflexivité et perspective en première personne : un apport décisif de la psychopathologie phénoménologique contemporaine à la compréhension de la schizophrénie ${ }^{\text {is }}$
}

Hyper reflexivity and the first-person perspective: A decisive contribution of contemporary phenomenological psychopathology to the understanding of schizophrenia

Jérôme Englebert (PhD) (Maître de conférences) $)^{\mathrm{a}, *, \mathrm{~b}}$, Pr Giovanni Stanghellini $(\mathrm{PhD})^{\mathrm{c}, \mathrm{d}}$, Caroline Valentiny (Psychologue) ${ }^{\mathrm{a}}$, Valérie Follet (Psychologue) ${ }^{\mathrm{a}}$, Pr Thomas Fuchs (PhD) ${ }^{\mathrm{e}}$, Pr Louis Sass $(\mathrm{PhD})^{\mathrm{f}}$

a Département de psychologie, université de Liège, bâtiment B33 Quartier-Agora, place des Orateurs 1, 4000 Liège, Belgique

${ }^{\mathrm{b}}$ Université de Lausanne, centre hospitalier universitaire vaudois (CHUV), 46, rue du Bugnon, 1011 Lausanne, Suisse

" Université "G. d'Annunzio" de Chieti, Via Dei Vestini, 66100 Chieti, Italie

"Université "Diego Portales" de Santiago, Calle Vergara 210, Santiago, Región Metropolitana, Chili

e Université de Heidelberg, Voss-Street 4, D-69115 Heidelberg, Allemagne

${ }^{\mathrm{f}}$ Université Rutgers, 152, Frelinghuysen Rd-Piscataway, 152, Frelinghuysen Rd, Piscataway, NJ 08854-8020, États-Unis

Reçu le 4 mars 2017

\section{Résumé}

Objectifs.-Cet article présente l'hypothèse, issue du champ de la psychopathologie phénoménologique, de l'hyper-réflexivité schizophrénique, selon laquelle les patients schizophrènes sont pris dans

\footnotetext{
گ̧ Toute référence à cet article doit porter mention : Englebert J, Stanghellini G, Valentiny C, Follet V, Fuchs T, Sass L. Hyper-réflexivité et perspective en première personne : un apport décisif de la psychopathologie phénoménologique contemporaine à la compréhension de la schizophrénie. Evol psychiatr XXXX; vol $\left(\mathrm{N}^{\circ}\right)$ : pages (pour la version papier) ou URL [date de consultation] (pour la version électronique).

* Auteur correspondant.

Adresse e-mail : jerome.englebert@ulg.ac.be (J. Englebert).
} 
l'expérimentation d'une conscience qui se retrouve contrainte d'interroger des phénomènes qui devraient aller intuitivement de soi.

Méthodes. - La perspective en première personne est une démarche qualitative complétant la lecture, traditionnelle dans le champ de la psychiatrie, en troisième personne - qui consiste en l'attribution depuis une position externe de signes cliniques repérés indépendamment du ressenti exprimé par le patient. Cette perspective en première personne concentre son attention sur l'expérience subjective qu'exprime le sujet. Le discours de plusieurs patients schizophrènes a été recueilli dans le cadre d'un dispositif clinique en utilisant l'échelle EASE (Examination of Anomalous Self-Experience). Cette échelle permet l'exploration semi-structurée, d'orientation phénoménologique, d'une série de difficultés révélatrices d'anomalies de l'expérience subjective.

Résultats. -En plus des phénomènes d'hyper-réflexivité, nos analyses mettent en évidence deux grandes caractéristiques de l'être-au-monde schizophrénique. D'abord une problématique du sens commun, qui pose la question de l'intersubjectivité. Ensuite l'existence d'un trouble de l'intercorporéité, les expériences d'hyper-réflexivité schizophrénique s'accompagnant d'une perte du « corps commun ».

Discussion. - L'application d'une perspective en première personne dans la compréhension du vécu des patients schizophrènes, ainsi que l'intérêt porté au phénomène d'hyper-réflexivité, permettent de proposer une vision de la schizophrénie qui n'est plus réduite à une lecture déficitaire de celle-ci (sans nier cette dernière), et qui découvre non pas un affaiblissement, mais une intensification de la conscience.

Conclusions. - La prise en considération de la dimension tacite de l'existence schizophrénique, telle que le suggère la psychopathologie phénoménologique contemporaine, offre de précieuses informations à la pratique clinique et suggère d'importantes perspectives de recherche associant aux designs méthodologiques conventionnels des perspectives novatrices et prometteuses.

(C) 2017 Elsevier Masson SAS. Tous droits réservés.

Mots clés : Schizophrénie ; Hyper-réflexivité ; Perspective en première personne ; EASE ; Intersubjectivité

\section{Abstract}

Objectives. - This paper considers the hypothesis of schizophrenic hyper reflexivity, which originates in the field of phenomenological psychopathology. It discusses the methodological context enabling these highly subjective manifestations to emerge. Starting from the seminal hypothesis developed by contemporary psychopathology that schizophrenic patients experience a form of consciousness that finds itself forced to question phenomena that should be intuitively self-evident, the idea of the present paper is to complete the usual interpretation of schizophrenia as a deficit by the hypothesis of an excessive functioning of consciousness.

Methods. - The first-person perspective is a qualitative approach completing the third-person interpretation, traditional in the field of psychiatry, which consists in the attribution, from an external position, of clinical signs noted independently from the perceptions expressed by the patient. The first-person perspective focuses on the patient's subjective experience as expressed by that individual. To understand schizophrenia according to this perspective, the narratives of several schizophrenic patients were collected in a clinical setting. We used the EASE scale (Examination of Anomalous Self-Experience) for this purpose. This scale offers a phenomenologically-oriented semi-structured exploration of a set of difficulties revealing anomalies in subjective experience, considered as self-awareness disturbances. EASE was developed on the basis of self-descriptions by patients suffering from schizophrenic spectrum disorders. This tool enables the coconstruction of a language between patient and clinician, focused on the patient's particular experiences and the unique phenomena he has to cope with.

Results. - Alongside the hyper reflexivity phenomena, the qualitative analyses of the descriptions collected highlight two important features of schizophrenic being-in-the-world. First a common sense issue, which raises the question of inter-subjectivity and the scope for schizophrenic patients to share in a social world. Secondly, the existence of an intercorporeality disturbance, since the schizophrenic hyper 
reflexivity experiences are accompanied by a loss of the "common body", the body being the scene where inter-subjectivity is played out.

Discussion. - The use of the EASE scale and the application of the first-person perspective provides a relevant psychotherapeutic approach, both in the process of acknowledgement of the illness (required from the patient as well as from the clinician), and in communication with the families of schizophrenic patients.

Conclusions. - The phenomenological interpretation provided by the application of a first-person perspective in the understanding of schizophrenic patients' experiences, and the inclusion of hyper reflexivity phenomenon, suggest perspectives on schizophrenia that are no longer reduced to a mere interpretation of deficit (without denying the deficit), showing not a weakening, but a surprising intensification of consciousness. (C) 2017 Elsevier Masson SAS. All rights reserved.

Keywords: Schizophrenia; Hyper reflexivity; First-person perspective; EASE; Inter-subjectivity

\section{Introduction}

Un apport crucial des analyses phénoménologiques en psychopathologie est de mettre en lumière que les personnes schizophrènes présentent une manière d'être caractérisée par une conscience fonctionnant selon des modalités inédites. La conscience, dans son acception phénoménologique, doit être envisagée comme la manière qu'a un sujet d'être en relation avec l'environnement, avec les autres et avec lui-même [1]. À la suite de travaux pionniers [2,3] et contemporains [1,4-8], de nombreuses données qualitatives permettent d'affirmer que les patients schizophrènes sont pris dans l'expérimentation d'une conscience qui se retrouve contrainte d'interroger des phénomènes qui devraient aller intuitivement de soi. Selon la célèbre formule d'Anna Rau, patiente de Blankenburg, le sujet schizophrène a perdu l'évidence naturelle des choses peuplant le monde. Il est essentiel de comprendre que cette modalité si particulière n'est pas à situer à un niveau purement cognitif ou intellectuel, mais s' ancre dans un mode d'être-au-monde plus profond touchant aux problématiques subjectives, corporelles et intersubjectives.

La prise en considération de cette dimension tacite de l'existence permet de compléter une lecture déficitaire de la schizophrénie — dominante notamment dans le paradigme cognitif par l'hypothèse d'un excès de fonctionnement de la conscience. Précisons, par ailleurs, que de nombreux travaux suggèrent une convergence d'évidence et une « homologie structurelle » entre les données empiriques des neurosciences, de la psychopathologie cognitive et de la neurobiologie et les descriptions cliniques et propositions théoriques de la psychopathologie phénoménologique [9-15]. La mise en évidence de ces corrélats ouvre d'importantes perspectives de recherche associant aux designs méthodologiques conventionnels des perspectives cliniques novatrices et prometteuses.

Bien que ces hypothèses soient régulièrement discutées dans le champ de la recherche internationale (voir par exemple 7 [16-18]), elles sont peu répercutées dans le champ de la psychopathologie francophone (on relèvera toutefois les excellentes synthèses suivantes : [19-21]). Sur les différences entre la psychopathologie phénoménologique anglo-saxonne et celle émergeant — sous l'égide notamment de l'École française de Daseinsanalyse — du contexte français, l'on se référera à [22]. L'objectif de cet article est de présenter cette hypothèse clinique décisive, révélée par une méthode inscrite dans une perspective en première personne, présentant des incidences sur la pratique psychothérapeutique, sur le processus de reconnaissance de la maladie, mais aussi sur le discours exprimé aux familles des patients à propos de la maladie. 


\section{Hyper-réflexivité et sens commun}

Depuis la psychanalyse notamment, il est incontestable que la conscience soit traversée, ou constituée, en plus de phénomènes explicites, par des analogons implicites. L'hypothèse de l'hyper-réflexivité ne suggère rien d'autre qu'un déséquilibre entre ces deux formes phénoménologiques de la conscience. Le sujet hyper-réflexif serait celui qui interroge sur un mode réflexif-explicite des phénomènes a priori préréflexifs-implicites ${ }^{1}$. Sans cesse colonisé par des éléments du monde ou de la pensée qui, dans le cas d'un rapport au monde fluide, se trouvent en arrière-plan, le schizophrène est entravé dans l'immédiateté de son existence. C'est là que se joue l'énigme schizophrénique :

« Je me demande des choses comme... un mot par exemple, la manière dont on compose un mot, comment ça se fait qu'on fait des mots comme ça, pourquoi c'est ces mots-là et pas des autres. Et pendant que j'y pense, la conversation tourne, elle tourne, et les autres me demandent un truc, mais moi je pense aux mots, et alors ça ramone, ça ramone, et finalement ça veut plus rien dire. Des fois je suis comme attrapé dans les questions, j'entends plus les autres. Après je marche dans la rue, je marche, et je suis dans les questions. Ça, ça me manque. Juste me promener sans m'inquiéter de rien quoi. »

«Le plus difficile c'est le doute, on doute sur toutes les idées et on se pose des questions à devenir fou, ça tourne à l'obsessionnel dans la tête. Il faut prendre distance et le regarder avec ironie... Le cerveau c'est sans arrêt des idées qui défilent, qui défilent. Comment voulez-vous que j'arrête de penser, même quand je suis comme un légume ça continue, c'est à la mort cérébrale que j'arrêterai de réfléchir. »

«Je ne veux plus deviner, ça me fatigue, parfois quelque chose tombe dans la tête, vous ne savez pas d'où ça vient, c'est épuisant, je dois tout le temps réfléchir sur ce qui se pond dans ma tête. »

Les séquences cliniques présentées dans cet article (apparaissant en italique) sont issues de notre pratique clinique. Une partie d'entre elles sont discutées dans Schizophrénie, conscience de soi, intersubjectivité [6]. Les discours ont été recueillis dans un dispositif clinique, au moyen de l'échelle EASE (outil dont nous discutons ci-après), auprès de sujets diagnostiqués schizophrènes.

Ces exemples cliniques suggèrent que l'hyper-réflexivité inspire intrinsèquement une convocation de tout le champ de la conscience. Comme la plupart des terminologies appliquées à la conscience ou à la vie subjective, le mot « réflexif » est ambigu. La réflexivité dans « l'hyperréflexivité » ne se réfère pas seulement aux formes volontaires ou intellectuelles de la conscience de soi (que l'on pourrait traduire par le terme anglais reflectivity), mais aussi à l'expérience d'anomalies plus spontanées de la conscience (se référant ici au mot anglais reflexivity). Dans une récente clarification [25], Sass propose, en s'inspirant de Merleau-Ponty, les termes français d' « hyper-réflexivité réfléchissante » pour qualifier la dimension volontaire et soumise à l'intention du sujet et d' « hyper-réflexivité opératoire » pour qualifier la dimension antéprédicative et non

\footnotetext{
1 Il est important de préciser que l'hyper-réflexivité n'est pas l'apanage des seuls patients schizophrènes. Avec Fuchs [16], nous pensons plutôt que, si de tels phénomènes se manifestent dans diverses pathologies, c'est toutefois dans la schizophrénie que l'on retrouve la dissolution la plus profonde de l'implicite et du « tenu-pour-acquis » du soi, des autres et du monde. Précisons également que plusieurs études mettent en évidence, pour des personnes en état d'introspection ou présentant un trouble de dépersonnalisation, des anomalies de l'expérience de soi analogues à celles retrouvées chez les personnes schizophrènes [23,24].
} 
intentionnelle (soumise à l'intentionnalité au sens de la philosophie phénoménologique). Précisons également qu'évoquer deux formes d'hyper-réflexivité (secondaire et primaire) doit être considéré comme une simplification heuristique, alors que la distinction réelle est sûrement progressive plutôt que dichotomique, et présente des variantes mixtes.

Ce principe d'une hyperconscience (plus générique et ontologique et incluant les deux formes d'hyper-réflexivité que nous venons d'objectiver) suggère, à l'appui de sérieux motifs cliniques, que la dimension subjective schizophrénique transcende le champ du réflexif et que c'est bien dans un mouvement de réverbération mettant en scène autrui que naît le paradoxe intersubjectif de la schizophrénie. Cette intrusion des phénomènes préréflexifs dans le champ de la conscience, en plus d'être centrale (structurale), a de nombreuses incidences ontologiques. La plus coûteuse d'entre elles se situe au niveau de la problématique du sens commun $[8,26]$. Plus qu'un défaut de sens - peut-être s'agit-il donc d'une compréhension exacte mais excessive —, la schizophrénie est un trouble de la dimension commune et relationnelle de la production de sens et de la diffusion de celui-ci. Le schizophrène manque l'échelon de l'intersubjectivité et, chemin faisant, se trouve « hors champ » de l'expérience commune. Les ingrédients nécessaires au partage social du sens sont absents. Dans une suite tautologique, l'hyper-réflexivité rend le sujet indisponible à l'aventure sociale.

«On se sent souvent à côté de la plaque, on n'est pas où on devrait être, on ne vit pas ce qu'on devrait vivre, on n'est pas dans le monde où on devrait vivre. On est à côté de la vie, on n'est pas dans la vie avec les autres. On est comme entre deux mondes, c'est difficile de réagir dans les situations sociales, on est parfois dans un monde, parfois dans l'autre. »

«Mon rapport particulier aux choses est tellement puissant que parfois ça m'empêche de vivre, j'ai parfois l'impression d'être trop conscient de tout ce que cela représente. Vivre est quelque chose qui est parfois trop compliqué, pas trop compliqué pour moi, mais trop compliqué pour l'existence. Je me pose des questions que la plupart des gens ne se posent pas, et cela me rend différent à leurs yeux, cela m'exclut peut-être un peu. »

L'on comprend ainsi que la perte de l'évidence naturelle ne se limite pas intrinsèquement au monde des ustensiles mais s'étend d'emblée à la possibilité d'un monde social. Immédiatement, le monde et ses possibilités d'interaction sont en train de se dérober : «On détache un grain de sable et toute la plage s'effondre » [27]. La catastrophe existentielle vécue par le sujet schizophrène ne résiderait donc pas dans la dimension cognitive des interrogations, mais bien dans la fracture sociale qui instantanément se révèle.

Précisons que c'est à Sass [28] que l'on doit la proposition princeps de l'hyper-réflexivité schizophrénique. Il est d'ailleurs intéressant de préciser que cette « découverte » est concomitante à une étude de l'esthétique et de la réflexivité moderne [28,29] et à une analyse des œuvres postmodernes (ou précurseurs) de Merleau-Ponty, Derrida et surtout Foucault. Sass identifie chez ce dernier, particulièrement dans Les mots et les choses [30], une analyse du statut problématique de la conscience de soi réflexive en révélant une épistémologie de l'acte de connaissance en tant qu'il est posé par le « sujet connaissant prenant conscience de son être propre en tant que perspective sur le monde » ([31], p. 99).

\section{Trouble de l'intercorporéité et de l'éprouvé émotionnel}

Si l'on se place du point de vue de la phénoménologie, la conscience schizophrénique accomplit peut-être un «fantasme » propre à la conscience, celui de se détacher du corps. Elle permet 
cette interrogation totale, solipsiste, démesurée, de sa propre phénoménalité. Toutefois, le paradoxe d'une conscience désincarnée serait au fond d'être réduite à cet objet d'intentionnalité. La conscience, dans sa quête de plénitude autoréflexive, perd la substance qui la constitue et devient un contenu sans contenant, opaque au monde.

Ces «paradoxes de la réflexivité » $[28,29,32]$, s'ils se jouent sur la scène du social, frappent le corps en ce qu'il a de plus profond et de plus enraciné. Car c'est bien le corps, et non l'esprit désincarné, qui domine la scène de l'intersubjectivité [33]. L'analyse philosophique de Merleau-Ponty [34] démontrait déjà de façon rigoureuse que l'intersubjectivité est une affaire d'intercorporéité. Cette proposition phénoménologique forte suggère qu'il existe, au-delà de l'hypothèse d'un esprit cognitif qui serait capable de s'associer au corps, une conscience incarnée (préréflexive) qui serait le médiateur décisif des trois aires de la vie psychique : autrui, l'environnement et soi-même. On peut en outre suggérer (sous forme d'hypothèse - a priori invérifiable) que les phénomènes d'hyper-réflexivité sont une conséquence d'un trouble fondamental de cette conscience sensorielle et primitive du soi. La syntonisation corporelle propre à tout usage social étant troublée, c'est bien une perte du « corps commun » qui accompagne les expériences d'hyper-réflexivité schizophrénique $[5,33,35,36]$. La pratique clinique révèle clairement que la conscience schizophrénique perçoit explicitement sa propre position excentrique par rapport à elle-même, au corps et au monde - c'est ce que l'on appelle la spatialisation de l'expérience corporelle ou le géométrisme morbide $[3,8]$.

«Ma vie consiste à penser. Je donne la priorité à réfléchir et cela prime sur mon implication dans les choses du quotidien. Je suis souvent en train d'observer ma propre existence. »

«J'avais la sensation bizarre de savoir où mon corps se trouvait en relation avec la pièce, et j'étais profondément conscient de mon poids et de l'espace rempli par mon corps. »

«Quand je dors, je sens que mon mental travaille fort, pense, comme si mon mental était en train de carburer d'une manière autonome, à côté de moi (montre de la main un espace à gauche de sa tête). »

«Souvent j'ai l'impression que le lieu de ma pensée se déplace dans mon corps. C'est parfois dans mon cerveau, parfois dans mes chevilles, parfois dans mon ventre. »

L'hyper-réflexivité consiste dès lors à poser comme objets explicites l'ensemble de sa propre existence, en ce compris, son propre corps ainsi que la rencontre avec le monde, qui généralement demeurent tacites et implicites. L'hyper-réflexivité est donc, en sa racine première, une crise de la conscience sensorielle de soi, un trouble du « moi minimal» ou «self de base » $[7,17]$ conduisant à l'éprouvé de corps «vécu » de façon fonctionnelle, impersonnelle et dévitalisée. Le corps schizophrénique tend vers la réalisation d'un destin d'objet technique, voire inerte, pour lequel l'aventure émotionnelle semble compromise.

\section{Incidence méthodologique : perspective en première personne}

La rencontre de l'hypothèse de l'hyper-réflexivité repose, d'un point de vue méthodologique, sur la prise en considération d'une perspective en première personne de l'expérience schizophrénique. Si la perspective en troisième personne repose sur l'attribution depuis une position externe de signes cliniques repérés indépendamment du ressenti exprimé par le patient (il s'agira notamment de l'objectivation — pourtant paradoxale — du délire et de l'hallucination du schizophrène), la démarche propre à la perspective en première personne concentre son 
attention sur l'expérience subjective qu'exprime le sujet. Ces signes cliniques, au premier rang desquels on retrouve l'hyper-réflexivité, révèlent l'expérience du soi et deviennent l'élément psychopathologique structurant de l'éprouvé schizophrénique.

Ce pas de côté méthodologique, reposant sur une démarche qualitative complétant une lecture en troisième personne, se révèle être un outil psychothérapeutique tout à fait pertinent. Il offre un accès à l'expression subjective du sujet schizophrène et ouvre la voie à une perspective intersubjective, co-construite. Cette perspective en «Je » permet également de désacraliser le problème de l'anosognosie et de repenser le processus de reconnaissance de la maladie (le mouvement de reconnaissance doit ainsi être réalisé également par le thérapeute), mais aussi de repenser le discours exprimé aux familles des patients concernant le trouble.

Des travaux initiés à Copenhague ont mené à la publication de l'échelle EASE (Examination of Anomalous Self-Experience) [37] dont la version francophone a été publiée dans L'Encéphale [38]. Citons également l'échelle EAWE (Examination of Anomalous World Experience) [39] qui a pour objectif d'analyser, au-delà de l'expérience du soi, les formes de rapports que le sujet entretient à son environnement (interrogeant le rapport à l'espace, au temps, à l'atmosphère notamment). Outre le fait de structurer la démarche du clinicien tout en respectant la dimension qualitative intrinsèque à cette pratique, cet outil offre l'apport décisif de permettre la co-construction d'un langage portant sur l'expérience particulière vécue par le patient et les phénomènes uniques qui le traversent. Les faire exister dans un espace partagé, au cœur d'une situation inédite, non seulement les circonscrit mais rend en quelque sorte légitimes ces expériences subjectives. Ces phénomènes trouvent alors une place dans l'expérience humaine. De façon parfois déconcertante, les patients semblent adhérer à ce « pari fou » consistant à leur rendre la parole :

«Ça m'a fait du bien, c'est bien de parler comme ça! Vous savez, elles ne sont pas bizarres vos questions. Enfin pas pour moi quoi. En fait, on ne m'a jamais demandé ces choses-là. Ça m'aide parce que les questions que vous vous posez, je me les pose aussi. »

« Je ne suis pas schizophrène, j'en ai toujours été convaincu. . . Mais, ce dont vous me parlez à travers vos questions, cette perte de l'évidence et cette hyper-attention aux choses du monde, la grande sensibilité dont nous parlons, cela correspond à ce que je vis. Si c'est ça la schizophrénie, je veux bien en rediscuter. »

L'échelle EASE, et de façon plus générale la considération en première personne propre au paradigme de la psychopathologie phénoménologique, se révèlent être un des alliés incontournables dans le processus de reconnaissance de la maladie. Cette perspective méthodologique permet de penser que pour qu'un tel processus émerge chez le patient, il faut qu'un autre processus se manifeste antérieurement chez son interlocuteur (en l'occurrence le clinicien). La reconnaissance de la maladie, si l'on se place dans une perspective en première personne, est, selon une logique inversée, celle qu'a d'abord à fournir le psychothérapeute.

\section{Conclusion}

Cet article avait pour objectif de présenter synthétiquement l'hypothèse phénoménologique de l'hyper-réflexivité schizophrénique et de discuter du contexte méthodologique permettant de faire émerger ces manifestations hautement subjectives. Cette proposition permet également de suggérer une compréhension de la schizophrénie qui n'est plus réduite uniquement à une lecture déficitaire (sans nier celle-ci), mais en lui découvrant un étonnant excès de réflexivité. Rejoignant l'intuition du poète, «Le fou n'est pas l'homme qui a perdu la raison. Le fou est celui qui a tout 
perdu, excepté la raison » ([40], p. 354), Sass s'interroge bien à raison : «Et si la folie, du moins dans certaines de ses formes, dérivait d'une intensification plutôt que d'un affaiblissement de la conscience, et d'une aliénation non pas de la raison mais des émotions, de l'instinct, du corps? » ([28], p. 4).

\section{Déclaration de liens d'intérêts}

\section{Les auteurs déclarent ne pas avoir de liens d'intérêts.}

\section{Références}

[1] Parnas J, Bovet P, Zahavi D. Schizophrenic autism: clinical phenomenology and pathogenetic implications. World Psychiatry 2002;1(3):131-6.

[2] Blankenburg W. La perte de l'évidence naturelle: une contribution à la psychopathologie des schizophrénies paucisymptomatiques (1971). Paris: PUF; 1991.

[3] Minkowski E. La schizophrénie (1927). Paris: Petite Bibliothèque Payot; 2002.

[4] Englebert J. Psychopathologie de l'homme en situation. Paris: Hermann; 2013.

[5] Englebert J, Valentiny C. Schizophrénie, conscience de soi, intersubjectivité : essai de psychopathologie phénoménologique en première personne. Bruxelles: De Boeck; 2017.

[6] Fuchs T. The tacit dimension. Philos Psychiatr Psychol 2001;8:323-6.

[7] Sass LA. Self-disturbance and schizophrenia: structure, specificity, pathogenesis (current issues, new directions). Schizophr Res 2014;152(1):5-11.

[8] Stanghellini G. Disembodied spirits and deanimated bodies: the psychopathology of common sense. Oxford: Oxford University Press; 2004.

[9] Fuchs T. Das Gehirn-ein Beziehungsorgan: eine phänomenologisch-ökologische Konzeption. Stuttgart: Kohlhammer; 2009.

[10] Fuchs T. Embodied cognitive neuroscience and its consequences for psychiatry. Poiesis Praxis 2009;6(3-4):219-33.

[11] Fuchs T. Phenomenology and psychopathology. In: Schmicking D, Gallagher S, editors. Handbook of phenomenology and cognitive science. Dordrecht: Springer; 2010. p. 546-73.

[12] Nelson B, Whitford TJ, Lavoie S, Sass LA. What are the neurocognitive correlates of basic self-disturbance in schizophrenia? Integrating phenomenology and neurocognition. Part 1 (Source monitoring deficits). Schizophr Res 2014;152(1):12-9.

[13] Nelson B, Whitford TJ, Lavoie S, Sass LA. What are the neurocognitive correlates of basic self-disturbance in schizophrenia? Integrating phenomenology and neurocognition: part 2 (aberrant salience). Schizophr Res 2014;152(1):20-7.

[14] Borda JP, Sass LA. Phenomenology and neurobiology of self disorder in schizophrenia: primary factors. Schizophr Res 2015;169:464-73.

[15] Sass LA, Borda JP. Phenomenology and neurobiology of self disorder in schizophrenia: Secondary factors. Schizophr Res 2015;169:474-82.

[16] Fuchs T. The psychopathology of hyperreflexivity. J Speculative Philos 2010;24(3):239-55.

[17] Nelson B, Parnas J, Sass L. Disturbance of minimal self (ipseity) in schizophrenia: clarification and current status. Schizophr Bull 2014;40(3):479-82.

[18] Stanghellini G, Rossi R. Pheno-phenotypes: a holistic approach to the psychopathology of schizophrenia. Curr Opin Psychiatry 2014;27(3):236-41.

[19] Martin B, Giersch A, Cermolacce M, Berna F, Dubreucq J, Franck N. Self minimal et schizophrénie. EMC Psychiatr 2016:1-12.

[20] Martin B, Piot MA. Approche phénoménologique de la schizophrénie. Info Psychiatr 2011;87:781-90.

[21] Chaperot C. Schizophrénie et condition humaine: abord daseinanalytique. Evol psychiatr 2015;80(1):55-70.

[22] Basso E. Où va la philosophie de la psychiatrie ? Rev Synth 2016;137(1-2):153-75.

[23] Sass LA, Pienkos E, Nelson B. Introspection and schizophrenia: a comparative investigation of anomalous selfexperiences. Conscious Cogn 2013;22:853-67.

[24] Sass LA, Pienkos E, Nelson B, Medford N. Anomalous self-experience in depersonalization and schizophrenia: a comparative investigation. Conscious Cogn 2013;22:430-1. 
[25] Sass LA. Preface to revised edition. In: Madness and Modernism: insanity in the light of modern art, literature and thought. Oxford: Oxford University Press; 2017 [s.p].

[26] Stanghellini G. At issue: vulnerability to schizophrenia and lack of common sense. Schizophr Bull 2000;26(4): 775-87.

[27] Michaux H, La, Ralentie. L'espace du dedans. Paris: Gallimard; 1937/1966. p. 216-25.

[28] Sass LA. Madness and modernism: insanity in the light of modern art, literature. In: and thought. New York: BasicBooks; 1992 [nouv. ed. rév. Oxford: Oxford University Press; 2017].

[29] Sass LA. Les paradoxes de la réflexivité. In: Englebert J, Cormann G, editors. Psychopathologie et philosophie : nouveaux débats et enjeux contemporains. Paris: Le Cercle Herméneutique; 2016. p. 26-7 [p. 185-219].

[30] Foucault M. Les mots et les choses. Paris: Gallimard; 1966.

[31] Sass LA. Foucault et l'autoréflexion moderne. Temps Mod 2009;656(5):99-143.

[32] Sass LA. Les paradoxes du délire: Wittgenstein, Schreber et l'esprit schizophrénique (1994). Paris: Ithaque; 2010 [traduit par P.-H. Castel].

[33] Stanghellini G. Embodiment schizophrenia. World Psychiatry 2009;8(1):56-9.

[34] Merleau-Ponty M. Phénoménologie de la perception. Paris: Gallimard; 1945.

[35] Englebert J, Gauthier JM. Géographie et psychose : territoire et perte de corps commun. Ann Med Psychol 2011;169:559-63.

[36] Englebert J, Valentiny C. Le schizophrène comme hyper-philosophe. In: Englebert J, Cormann G, editors. Psychopathologie et philosophie : nouveaux débats et enjeux contemporains. Paris: Le Cercle Herméneutique; 2016. p. 26-7 [p. 221-236].

[37] Parnas J, Møller P, Kircher T, Thalbitzer J, Jansson L, Handest P, et al. EASE: Examination of Anomalous SelfExperience. Psychopathology 2005;38(5):236-58.

[38] Parnas J, Møller P, Kircher T, Thalbitzer J, Jansson L, Handest P, et al. EASE : évaluation des anomalies de l'experience de soi. Encephale 2012;38:S121-45 [traduit par M. Cermolacce et P. Bovet].

[39] Sass LA, Pienkos E, Skodlar B, Stanghellini G, Fuchs T, Parnas J, et al. EAWE: Examination of Anomalous World Experience. Psychopathology 2017;50:10-54.

[40] Chesterton GK. Orthodoxie. Paris: Gallimard; 1908. p. 1984. 